Erratum

\title{
Variability of Prolactin Response to Intravenous and Intramuscular Haloperidol in Normal Adult Men
}

Psychopharmacology 61, 17-24 (1979)

On page 17, the authors' names should have appeared in the following order:

Sally E. Hays ${ }^{1,2}$ and Robert T. Rubin ${ }^{2}$

Neuro- and Biobehavioral Sciences Program, Stanford University School of Medicine, Stanford, California, U.S.A.

2 Department of Psychiatry, Harbor General/U.C.L.A. Medical Center, 1000 West Carson Street, Torrance, California, 90509, U.S.A. 